\title{
Introduction: thymus development and function in health and disease
}

\author{
Georg A. Holländer ${ }^{1,2,3}$ \\ Published online: 26 February 2021 \\ (C) Springer-Verlag GmbH Germany, part of Springer Nature 2021
}

The thymus provides throughout life the physiological site for the development of $\mathrm{T}$ lymphocytes. Hence, its function is critical for the successful establishment and maintenance of the immune system's capacity to distinguish between vital "Self" and injurious "Non-self". This essential competence is primarily instructed by thymic epithelial cells (TEC) and tissue-resident haematopoietic cells that constitute important components of the organ's stroma. Recent years have refined our understanding of the molecular and cellular mechanisms that control the formation, maintenance and function of the thymus. The series of reviews in this issue of Springer Seminars in Immunopathology provides a timely collection of scholarly reviews that give an account of the progress made in understanding the role of the thymus in health and disease.

The thymus was first recognized as an independent organ by the Greek and its name relates tellingly to a term in philosophy designating passion, soul, ardour and courage. The discovery of the tissue's essential role in the defence of infections and cancer was however only made in the sixties of the last century. Thus, the thymus was the last major human organ to reveal its intricate biological function. As so frequently in biomedical research, this discovery occurred following a fortuitous finding, namely that experimentally thymectomised mice lost weight and died prematurely after weaning. This seminal observation

This article is a contribution to the special issue on: The thymus and autoimmunity - Guest Editor: Georg Holländer

Georg A. Holländer

georg.hollander@paediatrics.ox.ac.uk

1 Department of Paediatrics and the Weatherall Institute of Molecular Medicine, University of Oxford, Oxford, UK

2 The University Children's Hospital of Basel and the Department of Biomedicine, University of Basel, Basel, Switzerland

3 Department of Biosystems Science and Engineering, ETH Zurich, Basel, Switzerland led JFAP Miller to the understated claim 'The thymus at birth may be essential for life' [1]. The review by V. Geenen, "The thymus and the science of Self" examines this assertion from a historical perspective investigating how our biological knowledge of the thymus has evolved over two millennia [2]. In addition, the author also offers an inspirational view on the organ's phylogenetic importance to maintain the survival of species that have adopted a complex multicellular existence.

The thymus generates multiple subsets of $T$ cells, each harnessed with individual functions relevant for the complex response to antigens. The review by E. Cosway and colleagues, "The Thymus Medulla And Its Control Of $\alpha \beta T$-cell Development", sheds light on the specific roles of the thymus medulla and especially its epithelial cells to create a specialized microenvironment that fosters the development and education of specialized $\mathrm{T}$ cell subsets prior to their exit to the periphery [3]. The authors describe the phenotypic and functional characteristics of previously identified as well as recently newly recognized thymic epithelia that form a complex scaffold creating tailored niches for specific functions. The review also highlights the molecular mechanisms so far defined that assure the final maturation and eventual exit of developing $\mathrm{T}$ cells (a.k.a. thymocytes) from the thymus. Unexpectedly, some of these processes seem to be largely independent of a normally arranged and correctly composed medullary TEC compartment.

The review by Deng and colleagues, "Leaving no one behind: tracing every human thymocyte by single cell RNAsequencing" presents a summary of recent exciting findings related to human intrathymic $\mathrm{T}$ cell development [4]. The authors discuss recent findings that have drawn on using single cell transcriptomics and advanced computational analyses to define human $\mathrm{T}$ cell ontogeny and to identify molecular mechanisms commanding individual cell fates. In addition, the article analyses the latest gene expression profiles that allowed the identification of novel TEC subtypes, thus further dissecting the complexity of the thymus stroma. For both 
lineages, the article highlights important differences between the human and mouse thymus, the latter being the experimental system so far most frequently employed to study thymus biology.

The $\mathrm{T}$ cell antigen receptor repertoire is initially generated during thymocyte development in a pseudo-random fashion. To maintain immunological tolerance to the body's own tissue antigens and to regulate antigen-specific responses of the adaptive immune system, the thymus employs several distinct strategies to achieve that vital distinction between "Self" and "Non-Self". One strategy involves the intrathymic depletion of thymocytes that express an antigen receptor with high affinity for self-antigens as these cells constitute a potential hazard eliciting autoimmunity. The review by Marx, "Thymus and autoimmunity", details the importance and molecular mechanisms of individual cellular stromal components to express most of the protein-coding genes. Known as promiscuous gene expression, this process enables the presentation of an extensive library of tissue-restricted antigens against which the $\mathrm{T}$ cell antigen receptors are probed [5].

Another strategy to maintain immunological tolerance to "Self" concerns the diversion of self-reactive $\mathrm{T}$ cells to a natural T regulatory cell (nTreg) fate. nTreg suppress the activation, expansion and effector functions of $\mathrm{T}$ cells stimulated in the context of an immune response to self as well as foreign antigens. The review by Bacchetta and Weinberg, "Thymic origins of autoimmunity - lessons from inborn errors of immunity", discusses in detail the intrathymic development of nTreg and examines the molecular mechanisms by which $\mathrm{nTreg}$ attain and exercise their specific functions [6]. Furthermore, the authors review the evidence of monogenic defects that compromise nTreg activity and present novel approaches to correct their deficiencies.

The article "Inborn errors of thymic stromal cell development and function" by Kreins and colleagues provides a detailed review of aetiologies that cause thymic stromal cell defects [7]. Genetic alterations that range from partial monosomy to a loss of function of single loci have been identified in humans as the molecular cause both of a complete or partial absence of thymic tissue and the origin of functional TEC deficits that compromise the generation and selection of naïve yet self-tolerant $\mathrm{T}$ cells. Given that the identification of these diseases can be challenging, the authors also discuss the principles underpinning their diagnosis and outline established as well as future therapies to regain thymus function.

In addition to inborn errors of lymphoid and TEC development, several other factors including chemotherapy, conditioning regimens in the context of allografts, graft-versushost disease and steroid therapy reduce thymus tissue mass and change the organ's cellular composition and architecture. Collectively, these alterations affect the generation, selection and output of naive $T$ cells. The review by Velardi and colleagues, "The role of the thymus in allogeneic bone marrow transplantation and the recovery of the peripheral T cell compartment" summarizes in detail the present understanding how thymus function is compromised by cytoablative conditioning and how these changes affect the reestablishment of a peripheral $\mathrm{T}$ cell compartment following haematopoietic stem cell engraftment [8]. The article also summarizes currently followed strategies and therapeutic advances that promote thymic reconstitution designed to fast-track immune reconstitution in transplant recipients.

The article by Granadier and colleagues, "Dynamics of thymus function and $T$ cell receptor repertoire breadth in health and disease" reviews the impact of both acute and chronic impairments of thymus function, such as stress and ageing, respectively [9]. Each of these insults has different cellular consequences and thus requires distinct therapeutic strategies to regain thymus function. The paper details the molecular mechanisms that control the remarkable capacity of the thymus for endogenous repair and how these provide a rationale for therapeutic regenerative interventions. Moreover, the authors discuss sensitive molecular tools and methods that allow the qualitative and quantitative assessment of thymus function.

The last article in this series, "The contribution of thymic tolerance to central nervous system autoimmunity" reviews current knowledge concerning a so far rather unappreciated role of the thymus in the pathogenesis of autoimmune diseases affecting the central nervous system (CNS) [10]. Here, Alberti and Handel discuss the experimental and clinical evidence for pathogenic thymic mechanisms that underpin these conditions. The authors also identify potential susceptibility factors that link thymus function to CNS autoimmunity and examine new options for the treatment of these pathologies.

Collectively, we hope that these articles offer an up-to-date insight into thymus function in health and disease. They should also provide convincing scientific evidence to dispute the conviction of Sir Peter Medawar postulated in 1963 that "We shall come to regard the presence of lymphocytes in the thymus as an evolutionary accident of no very great significance" [11].

\section{References}

1. Miller JF (1961) Analysis of the thymus influence in leukaemogenesis. Nature 191:248-249

2. Geenen V (2021) The thymus and the science of self. Semin Immunopathol. https://doi.org/10.1007/s00281-020-00831-y

3. Cosway EJ, James KD, Lucas B, Anderson G, White AJ (2020) The thymus medulla and its control of $\alpha \beta$ T cell development. Semin Immunopathol. https://doi.org/10.1007/s00281-020-00830-Z

4. Deng Y, Chen H, Zeng Y, Wang K, Zhang H, Hu H (2021) Leaving no one behind: tracing every human thymocyte by single cell RNAsequencing. Semin Immunopathol. https://doi.org/10.1007/s00281020-00834-9 
5. Marx A, Yamada Y, Simon-Keller K, Schalke B, Willcox N, Ströbel P, Weis CA (2021). Thymus and autoimmunity. Semin Immunopathol. https://doi.org/10.1007/s00281-02100842-3

6. Bacchetta R, Weinberg K (2021) Thymic origins of autoimmunity - lessons from inborn errors of immunity. Semin Immunopathol. https://doi.org/10.1007/s00281-020-00835-8

7. Kreins AY, Maio S, Dhalla F (2020) Inborn errors of thymic stromal cell development and function. Semin Immunopathol. https:// doi.org/10.1007/s00281-020-00826-9

8. Velardi E, Clave E, Arruda LCM, Benini F, Locatelli F, Toubert A (2021) The role of the thymus in allogeneic bone marrow transplantation and the recovery of the peripheral T-cell compartment. Semin Immunopathol. https://doi.org/10.1007/s00281-02000828-7
9. Granadier D, Iovino L, Kinsella S, Dudakov JA (2021) Dynamics of thymus function and $\mathrm{T}$ cell receptor repertoire breadth in health and disease. Semin Immunopathol. https://doi.org/10.1007/s00281021-00840-5

10. Alberti P, Handel AE (2020) The contribution of thymic tolerance to central nervous system autoimmunity. Semin Immunopathol. https://doi.org/10.1007/s00281-020-00822-Z

11. Medawar PB (1963) Discussion after Miller JFAP and Osoba D.; role of the thymus in the origin of immune competence. In: Westenholme GEW, Knight J (eds) The immunologically competent cell: its nature and origin. Ciba Foundation Study Group, London, $\mathrm{p} 70$

Publisher's note Springer Nature remains neutral with regard to jurisdictional claims in published maps and institutional affiliations. 